Proceedings of the International Symposium on Physics of Materials (ISPMA 14), September 10-15, 2017, Prague

\title{
Influence of Secondary Phase on Intrinsic Threshold and Path of Shear-Mode Fatigue Cracks in Metals
}

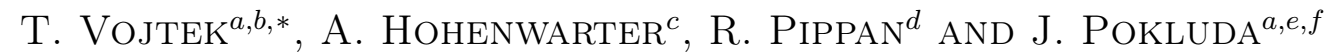

${ }^{a}$ Central European Institute of Technology (CEITEC), Brno University of Technology,

Purkyňova 123, 61200 Brno, Czech Republic

${ }^{b}$ CEITEC IPM, Institute of Physics of Materials, Academy of Sciences of the Czech Republic, Žižkova 22, 61662 Brno, Czech Republic

${ }^{c}$ Department of Materials Physics, Montanuniversität Leoben, Jahnstr. 12, A-8700 Leoben, Austria

${ }^{d}$ Erich Schmid Institute of Materials Science, Austrian Academy of Sciences, Jahnstr. 12, A-8700 Leoben, Austria

${ }^{e}$ Faculty of Mechanical Engineering, Brno University of Technology, Technická 2, CZ-61669 Brno, Czech Republic

${ }^{f}$ Faculty of Special Technology, Alexander Dubcek University of Trencin,

Pri parku 19, 91106 Trenčín, Slovak Republic

The paper deals with intrinsic behaviour of long fatigue cracks under the remote mode II and mode III loading, particularly with the intrinsic (effective) thresholds and the crack path geometry from the point of view of the competition between the local shear and the local opening growth mode. Unlike in the case of opening mode I cracks, the microstructure can significantly influence the dominant growth mechanism of the shear-mode cracks in dependence of the type of the secondary phase. The cementite lamellae of the pearlitic steel change the local growth mode from mode II to mode I and increased the effective mode II and mode III thresholds, whereas the $\beta$-Ti lamellae of the Ti6Al4V alloy do not change the intrinsic behaviour. The difference can be explained by different ability of dislocations to cross the phase boundary. The competition between the local shear and the local opening growth modes in metals is assessed by a newly proposed mode I branching criterion based on fracture mechanics. Using this criterion, the value of the deflection angle corresponding to the transition between the dominant mode I and dominant mode II crack growth was found to be of $46^{\circ}$. This result agrees well with the previously published value of the transient angle based on identification of characteristic fractographical patterns on the fracture surfaces of investigated metallic materials.

DOI: 10.12693/APhysPolA.134.699

PACS/topics: $62.20 . \mathrm{M}-$, 81.40.Np

\section{Introduction}

The fatigue crack growth threshold is one of important material characteristics, determining whether the long fatigue crack propagates or not. The resistance to fatigue crack growth should be divided into two components. The intrinsic component, also called the effective crack driving force, is responsible for cyclic plastic deformation of the crack tip leading to crack propagation. The extrinsic component represents crack tip shielding and is mostly realised behind the crack tip, for example, by different types of crack closure. In the near-threshold regime, the extrinsic component is often higher than the intrinsic one. Besides of being on the safe side, the intrinsic threshold data enable us a straightforward comparison with (and verification of) physical models of mechanisms of fatigue crack propagation.

Under mode I loading the intrinsic (effective) thresholds are well predictable and have approximately the same value for a particular metal independent of its microstructure. This value depends only on basic characteristics of the metal, the Young modulus $E$ and magnitude of the Burgers vector $b$ and,

\footnotetext{
* corresponding author; e-mail:

tomas.vojtek@ceitec.vutbr.cz
}

for single-phase metals, the following relationship [1] was proposed for the effective mode I threshold $\Delta K_{\text {Ieff,th: }}$ :

$$
\Delta K_{\text {Ieff,th }}=\frac{3}{4} E \sqrt{b} .
$$

In the case of shear-mode cracks, however, the effective thresholds depend on the crystal lattice type and microstructure. In previous studies (e.g. $[1,2]$ it was shown how the crystal lattice type of single-phase metals influences the local growth mechanism, which in turns influences the effective threshold.

The mechanism related to dominant mode I (opening) crack growth can be either ductile blunting or quasibrittle tearing, whereas that related to dominant mode II (shear) growth is shear along slip planes more or less parallel with the plane of the maximum shear stress (see [2] for more detail). The tendency to grow under local opening or local shear mode is reflected by the mean angle of deflection of the remote mode II loaded crack from the original precrack plane (the maximum shear stress plane). The following formulae [3], proposed for the prediction of the effective mode II threshold, are functions of the deflection angle $\alpha$ :

$$
\begin{aligned}
\Delta K_{\mathrm{II} \text { eff,th }}^{\text {theor I }} & =\frac{E \sqrt{b}}{2 \cos \frac{\alpha}{2} \sin \alpha}, \\
\Delta K_{\mathrm{II} \text { eff } \text { th }}^{\text {theor II }} & =\frac{G \sqrt{b}}{\frac{1}{2} \cos \frac{\alpha}{2}(3 \cos \alpha-1)} .
\end{aligned}
$$


For materials with dominant local mode I growth Eq. (2) should be used while Eq. (3) is for materials with dominant local mode II growth. Only the selected (dominant) mode is considered to contribute to crack propagation and the second (non-dominant) one can be neglected, which represents a new approach to mixed mode loading, different from the classical energetical summation of contributions from each of the modes I, II, and III.

It was shown that the typical angles $\alpha$ for pure bcc, hcp, and fcc metals are $15^{\circ}, 35^{\circ}, 50^{\circ}-65^{\circ}$, respectively. In bcc metals the shear-mode cracks grew under local mode II mechanism [3] due to high density of available slip planes in the lattice which enabled dislocation motion along the remote shear stress direction. This mechanism is very easy and energetically most convenient which is reflected by lower mode II effective thresholds than those of mode I in the bcc and hcp metals [3] (see also Table I).
Since most of the engineering materials are of multiphase structure, the purpose of this paper is to show the dependence of local growth mechanism of shear-mode cracks on the type of the secondary phase. Unlike in the case of mode I effective thresholds, the secondary phase can significantly change the mode II and mode III thresholds due to a change of the growth mechanism. Indeed, the previously published results [4] showed that the intrinsic behaviour of the ferritic-pearlitic steel was very different from that of the pure ferritic steel. Here, the influence of secondary phase type is studied also for the fully pearlitic steel and the Ti6Al4V titanium alloy in more detail. Based on fracture mechanics, a new criterion for mode I crack-branching is formulated and applied to all single- and dual phase metallic materials investigated hitherto.

Comparison of effective thresholds and local crack growth direction of remote mode II and mode III loading for both ferritic and pearlitic steels as well as for the pure $\alpha$-Ti and the $\alpha+\beta$ titanium alloy.

\begin{tabular}{|c|c|c|c|c|c|c|}
\hline \multirow{2}{*}{\multicolumn{3}{|c|}{ Material characteristic }} & \multicolumn{2}{|c|}{ Steels } & \multicolumn{2}{|c|}{ Titanium alloys } \\
\hline & & & \multirow{2}{*}{$\begin{array}{c}\text { ferritic steel } \\
2.5 \\
2.7^{a}\end{array}$} & \multirow{2}{*}{$\begin{array}{c}\text { pearlitic steel } \\
2.5 \\
3.0^{d}\end{array}$} & \multirow{2}{*}{$\begin{array}{c}\text { pure } \alpha \mathrm{Ti} \\
2.0^{e} \\
2.0^{e}\end{array}$} & \multirow{2}{*}{$\begin{array}{c}\alpha+\beta \mathrm{Ti} \\
2.0 \\
2.2^{e}\end{array}$} \\
\hline effective & mode I & $\begin{array}{l}\text { Eq. (1) } \\
\text { experimental }\end{array}$ & & & & \\
\hline $\begin{array}{l}\text { threshold } \\
{\left[\mathrm{MPa} \mathrm{m}^{1 / 2}\right]}\end{array}$ & $\begin{array}{l}\text { mode II } \\
\text { mode III }\end{array}$ & $\begin{array}{l}\text { Eqs. }(2),(3) \\
\text { experimental } \\
\text { experimental }\end{array}$ & $\begin{array}{c}1.4 \\
1.5^{b} \\
2.6^{c}\end{array}$ & $\begin{array}{c}2.2 \\
2.8^{b} \\
4.5^{c}\end{array}$ & $\begin{array}{c}1.5 \\
1.7^{b} \\
2.8^{c}\end{array}$ & $\begin{array}{c}1.5 \\
1.8^{b} \\
3.1^{f}\end{array}$ \\
\hline local crack & \multicolumn{2}{|c|}{$\begin{array}{l}\text { deflection of mode II cracks } \\
\left(70^{\circ} \text { for pure local mode I) }\right.\end{array}$} & $19^{\circ b}$ & $60^{\circ b}$ & $33^{\circ b}$ & $30^{\circ b}$ \\
\hline $\begin{array}{l}\text { growth } \\
\text { direction }\end{array}$ & \multicolumn{2}{|c|}{$\begin{array}{l}\text { twist of mode III cracks } \\
\left(45^{\circ} \text { for pure local mode } \mathrm{I}\right)\end{array}$} & $16^{\circ c}$ & $41^{\circ c}$ & $35^{\circ c}$ & $32^{\circ}$ \\
\hline \multicolumn{3}{|c|}{$\begin{array}{l}\text { dominant crack growth mechanism } \\
\text { under remote shear-mode loading }\end{array}$} & local mode II & local mode I & local mode II & local mode II \\
\hline
\end{tabular}

\section{Materials}

Two alloys with high importance in industrial applications were chosen for investigation. The first material was a titanium alloy ASTM grade 5 (Ti6Al4V) hardened by the $\beta$-Ti phase. The second was a fully pearlitic steel which is, for example, used for the production of rails. The rolling contact fatigue behaviour during the rail-wheel contact of rails is a practical example of shearmode dominant crack propagation.

The influence of the $\beta$-Ti lamellas in the Ti6Al4V alloy was compared to the influence of cementite lamellas in the pearlitic steel. Figure 1 shows the electron backscattered diffraction (EBSD) image of the Ti6Al4V alloy where the presence of the $\beta$-Ti phase in the form of lamellas is indicated by thin red volume inside the black phase boundaries.

\section{Experiments}

Two types of shear-mode tests were conducted at the stress ratio of $R=0.1$. First, cylindrical specimens with circumferential notch were loaded in torsion to achieve pure mode III loading and, second, a special device for mixed mode II + III loading was used to load the same specimens in simple shear. Combinations of mode II and mode III loading were present at the crack tip depending on the position around the circular cross-section, including pure mode II and pure mode III loading. A detailed description of the experimental arrangement can be found in $[1,2]$. Before the shear-mode tests precracks were generated at the notch root by cyclic compressioncompression loading $[5,6]$. Such created precracks were open due to residual opening stress and enabled measurements under closure-free conditions. No interaction of fracture surfaces (sliding-mode crack closure) was present at the beginning of the experiment, which allowed studying of the intrinsic behaviour. After precracking, the specimens were annealed in vacuum in order to eliminate the plastically deformed material state ahead of the crack tip and to avoid production of oxide on the fracture surfaces. The ARMCO iron was annealed at $950^{\circ} \mathrm{C}$ for $90 \mathrm{~min}$ and the resulting mean grain size was $110 \mu \mathrm{m}$. The corresponding metallography cut image can be found in [2]. The pearlitic steel was annealed at $850^{\circ} \mathrm{C}$ for 90 min (metallography cut in [7]). Commer- 


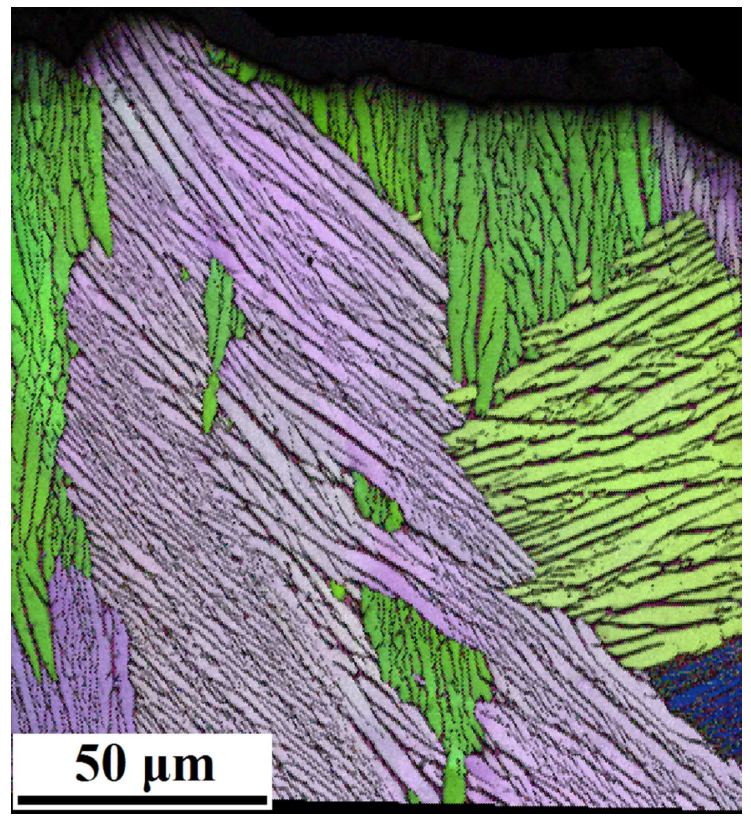

Fig. 1. EBSD image of the Ti6Al4V alloy with thin lamellae of the $\beta$-Ti phase. The cut includes the fracture surface of a remote mode II loaded crack on the top of the image.

cially pure titanium was annealed at $850^{\circ} \mathrm{C}$ for 90 min with the resulting mean grain size of $70 \mu \mathrm{m}$ (metallography cut in [1]). The $\mathrm{Ti}-6 \mathrm{Al}-4 \mathrm{~V}$ alloy was annealed at $1020^{\circ} \mathrm{C}$ for $60 \mathrm{~min}$ (metallography cut in [8]).

After application of $10^{5}$ loading cycles of the shearmode loading the experiment was stopped and the specimens were fractured by cyclic mode I loading. This allowed us a detailed fractographical observation of non-damaged fracture surfaces revealing fine patterns connected to crystallography and microstructure which would be smeared out if the crack propagated longer. The extent of the shear-mode crack propagation was measured in scanning electron microscope (SEM) allowing a determination of the effective threshold values by identification of the first specimen showing the crack advance.

The fracture surfaces were reconstructed in $3 \mathrm{D}$ by the stereophotogrammetry in SEM [9]. The height profile for measurement of the deflection angles is depicted in Fig. 2 (below the fracture surface). For the remote mode III loaded cracks the twist angles were also measured. The averaged deflection and twist angles are included in Table I that summarizes theoretical and experimental values for the two investigated materials and provides a comparison with those previously published for pure metals.

\section{Discussion}

The results revealed that the intrinsic behaviour (effective threshold and crack growth direction) of the Ti6Al4V alloy was nearly the same as that of the pure titanium. The lamellas of the $\beta$ phase in the Ti6Al4V alloy have the bcc lattice and create a coherent phase interface allowing slip transfer across the $\alpha-\beta-\alpha$ colony [14]. This
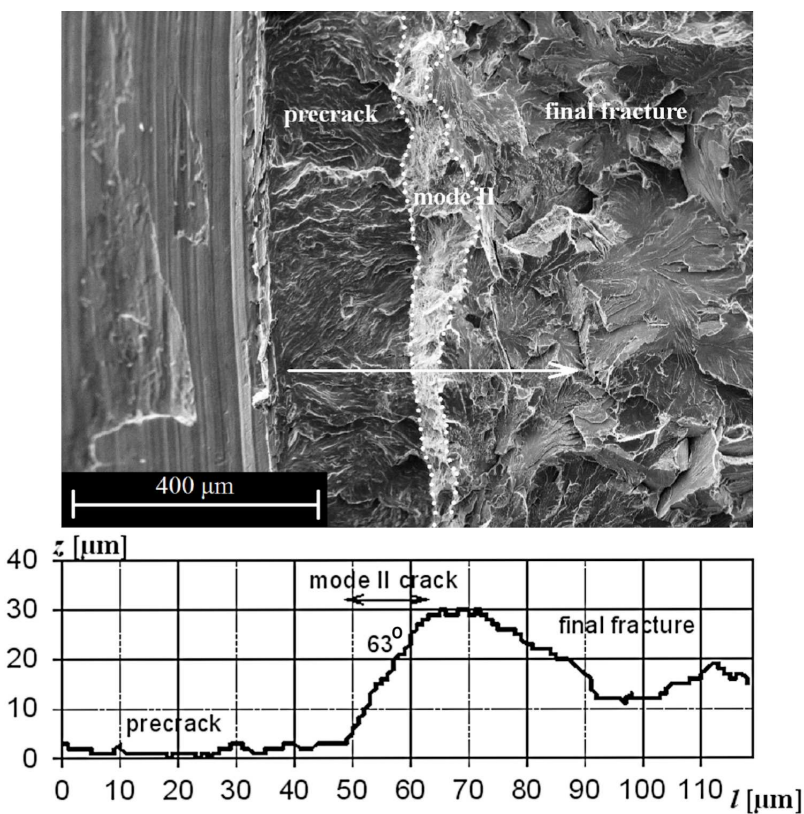

Fig. 2. Example of a fracture surface of a mode II crack in pearlitic steel. The height profile below is defined by the white arrow on the fracture surface. The deflection and twist angles in the pearlitic steel were much higher than those in ferritic steel, while in Ti6Al4V they were nearly equal to those of pure titanium.

corresponds to our observations as documented by the EBSD image in Fig. 2 which shows that the position of $\beta$ lamellas did not influence the crack growth direction across the primary $\alpha$-grains. Thus, the presence of $\beta$ Ti lamellas did not interfere with the shear-mode crack propagation which did not differ from that observed in the pure $\alpha$-Ti (see Table I).

On the other hand, the cementite-ferrite interface in the pearlitic steel represented a non-coherent boundary. It was found [15] that the slip transfer through this interface is possible only for half of the slip systems $1 / 2\langle 111\rangle\{110\}$ and for quarter of the slip systems $1 / 2\langle 111\rangle\{112\}$ of ferrite. Other slip systems do not cross the interface and are involved in the hardening of the ferrite phase of pearlite. Thus, the single-slip mechanism of the local mode II, characteristic for single-phase bcc metals, could not proceed across the cementite lamellae which resulted in the deflections along the lamella interfaces at many sites of the precrack front. These deflections caused a change to the local mode I mechanism corresponding to a higher effective threshold.

As already mentioned, the determination of the dominant local loading mode is important for the theoretical prediction of effective shear-mode fatigue threshold values. For this reason, Eqs. (2) and (3) can be simply used to formulate a new criterion which clearly defines the upper limit of the branching angle associated with dominant mode II crack growth, the so-called transition angle $\alpha_{t}$. At this angle, the effective mode II thresholds given by Eqs. (2) and (3) are identical, i.e. 
$\Delta K_{\mathrm{II} \mathrm{eff,th}}^{\text {theor }}=\Delta K_{\mathrm{II} \mathrm{eff,th}}^{\text {theor II }}$. The angles $\alpha<\alpha_{t}$ correspond to values of $\Delta K_{\text {II eff,th }}^{\text {theor II }}$ lower than those of $\Delta K_{\text {II eff,th }}^{\text {theor I }}$ which means the dominant mode II growth (and vice a versa for angles $\alpha>\alpha_{t}$ related to mode I dominance). Equating relationships (2) and (3) gives

$$
E\left(3 \cos \alpha_{t}-1\right)=4 G \sin \alpha_{t},
$$

which, for the Poisson ratio $\nu=0.3$, leads to

$$
\begin{aligned}
& \cos \alpha_{t}=\frac{1}{3}\left(\frac{20}{13} \sin \alpha_{t}+1\right), \\
& \alpha_{t}=2 \arctan \left(\frac{1}{26} \sqrt{438}-10\right) \cong 45.6^{\circ} .
\end{aligned}
$$

The result $\alpha_{t} \approx 46^{\circ}$ is sufficiently accurate for all metallic materials. According to their deflection angles (see Table I) the cracks in ferritic steel as well as in both titanium grades grow under the dominant mode II mechanism $\left(\alpha<\alpha_{t}\right)$, while those in the pearlitic steel grow in the dominant mode I mechanism $\left(\alpha>\alpha_{t}\right)$.

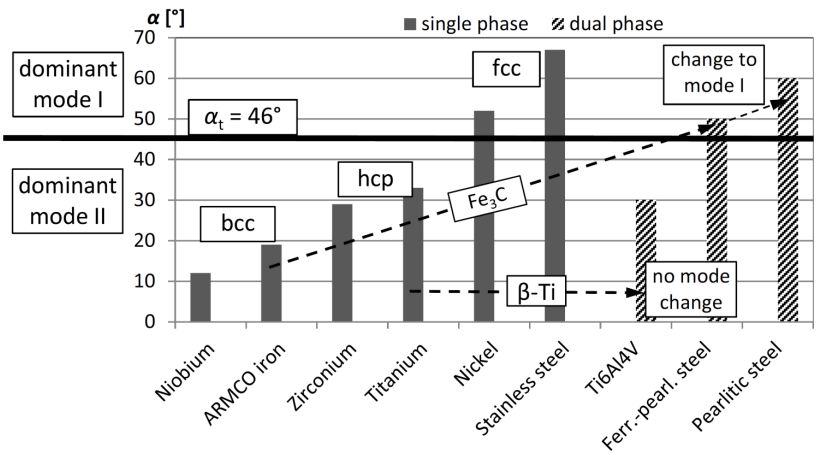

Fig. 3. Illustration of the types of dominant growth mechanisms in single-phase alloys (full bars) and in dual-phase materials (shaded bars).

Let us emphasize that, in the previous studies $[3,16]$, the dominant growth mode was determined by identification of characteristic fractographical patterns on the fracture surfaces of investigated materials. This analysis led to a value $\alpha_{t}$ in between 40 and 50 degrees, in a good agreement with $\alpha_{t} \approx 46^{\circ}$, determined by the new criterion. In Fig. 3 the bar chart illustrates the types of dominant growth mechanism in single-phase materials (full bars) as well as in dual-phase alloys (shaded bars). One can see that bcc and hcp single-phase metals exhibit a dominant mode II crack growth while the mode I growth dominates in fcc single-phase metals. The crystallographic reasons for such a kind of branching behaviour are explained elsewhere [1]. The arrows in Fig. 3 indicate how the type of secondary phase changes the branching behaviour of the matrix, as already discussed in this section.

\section{Conclusions}

This work studied the influence of type of the secondary phase on the dominant growth mechanism of fatigue cracks under the remote shear mode loading in metallic materials. It was shown that, in the pearlitic steel, the presence of cementite lamellae caused a change of the local mode II growth mechanism into the local mode I mechanism. Consequently, the effective mode II and mode III thresholds increased in comparison with the purely ferritic steel. On the other hand, a rather coherent phase boundary between the $\alpha$-titanium (hcp) and the $\beta$-Ti lamellae (bcc) in the Ti6Al4V alloy allowed for dislocation crossing and did not change the local growth mechanism. Thus, the effective mode II and mode III thresholds in this alloy remained approximately equal to those previously measured for the pure $\alpha$-titanium. The competition between the local shear and the local opening growth mode was classified by a newly proposed mode I branching criterion based on fracture mechanics. The value of the transition deflection angle determined by this criterion $\left(\alpha_{t} \approx 46^{\circ}\right)$ agrees well with the previously published result based on identification of characteristic fractographical patterns on the fracture surfaces of metallic materials ( $\alpha_{t}$ in between 40 and 50 degrees).

\section{Acknowledgments}

The authors acknowledge the financial support of this work by the Czech Science Foundation (GA CR) in the frame of the Project No. 17-15716Y, by the Slovak Research and Development Agency in the frame of the grant APVV-15-0710 and by the Ministry of Education, Youth and Sports of the Czech Republic under the project CEITEC 2020 (LQ1601).

\section{References}

[1] J. Pokluda, R. Pippan, T. Vojtek, A. Hohenwarter, Fatigue Fract. Eng. Mater. Struct. 37, 232 (2014).

[2] T. Vojtek, R. Pippan, A. Hohenwarter, L. Holáň, J. Pokluda, Acta Mater. 61, 4625 (2013).

[3] T. Vojtek, R. Pippan, A. Hohenwarter, J. Pokluda, Eng. Fract. Mech. 174, 117 (2017).

[4] T. Vojtek, J. Pokluda, A. Hohenwarter, R. Pippan, Acta Phys. Pol. A 128, 611 (2015).

[5] R. Pippan, L. Plöchl, F. Klanner, H.P. Stüwe, ASTM J. Test. Eval. 22, 98 (1994).

[6] J.C. Newman, Y. Yamada, Int. J. Fatigue 32, 879 (2010).

[7] J. Pokluda, T. Vojtek, A. Hohenwarter, R. Pippan, Fract. Struct. Integrity 34, 142 (2015).

[8] T. Vojtek, J. Pokluda, A. Hohenwarter, R. Pippan, Solid State Phenom. 258, 265 (2017).

[9] J. Stampfl, S. Scherer, M. Gruber, O. Kolednik, Appl. Phys. A 63, 341 (1996).

[10] R. Pippan, Mater. Sci. Eng. A 138, 1 (1991).

[11] T. Billaudeau, Y. Nadot, G. Bezine, Acta Mater. 52 , 3911 (2004).

[12] B.L. Boyce, R.O. Ritchie, Eng. Fract. Mech. 68, 129 (2001).

[13] T. Vojtek, S. Žák, J. Pokluda, Int. J. Fatigue 115, 35 (2018).

[14] Z. Zhang, T.-S. Jun, T.B. Britton, F.P.E. Dunne, J. Mech. Phys. Solids 95, 393 (2016).

[15] L.E. Karkina, I.N. Karkin, I.G. Kabanova, A.R. Kuznetsov, J. Appl. Crystallogr. 48, 97 (2015).

[16] T. Vojtek, A. Hohenwarter, R. Pippan, J. Pokluda, Int. J. Fatigue 92, 470 (2016). 